\title{
Microflow Extraction Using a Microchip Incorporating Microchannels Based on the Tube Radial Distribution Phenomenon
}

\author{
Naomichi SUZUKI ${ }^{1}$, Masahiko HASHIMOTO ${ }^{1}$ and Kazuhiko TSUKAGOSHI ${ }^{1,2} *$ \\ ${ }^{1}$ Department of Chemical Engineering and Materials Science, Faculty of Science and Engineering, \\ Doshisha University, Kyotanabe, Kyoto 610-0321, Japan \\ ${ }^{2}$ Tube Radial Distribution Phenomenon Research Center, Doshisha University, Kyotanabe, Kyoto \\ 610-0321, Japan
}

(Received March 4, 2015; Accepted November 2, 2015)

\begin{abstract}
When homogeneous solutions that feature two-phase separation properties, such as a ternary mixed solvent solution of water-acetonitrile-ethyl acetate, are fed into a microspace, such as a capillary tube and a microchannel, the solvent molecules are radially distributed into the microspace, generating inner and outer phases. This is called "tube radial distribution phenomenon" (TRDP). In this study, microflow extraction for the Fe(III)-8-hydroxyquinoline complex was carried out using a microchip incorporating microchannels based on the TRDP. A microchip in which one wide channel was separated into three narrow channels was designed and manufactured. When the ternary mixed solvent solutions of water (acetic acid aqueous solution, pH3.5)-acetonitrile-ethyl acetate (volume ratio 40:45:15) containing $\mathrm{Fe}(\mathrm{III})$ and 8-hydroxyquinoline were fed into the wide channel under laminar flow conditions, the solvent molecules were radially distributed in the channel, generating inner (organic solvent-rich) and outer (water-rich) phases. The Fe(III)-8-hydroxyquinoline complex in the carrier solution was distributed between the inner and outer phases due to its hydrophilic nature, and then collected through the three narrow channels. The concentration of $\mathrm{Fe}$ (III) in the center narrow channel was greater than those in the two outer narrow channels through extraction with 8-hydroxyquinoline.
\end{abstract}

\section{Introduction}

Micro-total analysis ( $\mu$-TAS) systems that include microfluidic device technology are a useful and interesting aspect of analytical chemistry. Microfluidics exhibit various types of fluidic solvent behavior in a microspace. This microfluidic behavior has been studied by varying the flow rate of the solvents, by using aqueous-organic mixed solvent solutions, and/or by introducing specific obstacles into the microspace $[1,2]$. The fluidic behavior of the solvents in the microspace is really related to the separation, mixing, diffusion, and reaction of the solutes. Extraction is one of the most useful and effective separation methods. Recently, microflow solvent extraction systems have also been investigated by using a liquid-liquid interface between an aqueous and organic solvent solution in the microspace [3,4]. Microflow solvent extraction has several advantages over conventional macroscale solvent extraction. For instance, the microflow system has a larger interfacial area per unit volume than that of the conventional macroscale flowsystem, leading to an enhancement of extraction efficiency.

When homogeneous solutions that feature a two-phase separation property, such as a water-acetonitrile-ethyl acetate mixed solvent solution, are delivered into a microspace, such as capillary tubes and microchannels in a microchip, the solvent molecules are radially distributed in the microspace 
under laminar flow conditions. This is called the "tube radial distribution phenomenon" (TRDP) [5-7]. For example, in the TRDP with a ternary mixed solvent solution of organic solvent-rich, through the two-phase separation property, an organic solvent-rich phase is formed around the middle of the microspace as an inner phase, while a water-rich phase is formed near the inner wall as an outer phase. The TRDP creates a specific liquid-liquid interface in the microspace to provide inner and outer phases.

We reported the extraction of $\mathrm{Fe}(\mathrm{III})$ through 8-hydroxyquinoline complex formation with the ternary mixed solvent solution, water (acetic acid aqueous solution, $\mathrm{pH} 3.5$ )-acetonitrile-ethyl acetate, in a batch-type reactor in our previous paper [8]. Also, we examined the distribution behavior of $\mathrm{Cu}$ (II) in the TRDP with the ternary mixed solvent solution in the microchannels on a microchip without any compexing agents [9]. In this study, the extraction of Fe(III)-8-hydroxyquinoline complex with the TRDP was carried out with a microflow system using a microchip incorporating triple-branch microchannels. We call the extraction procedure "tube radial distribution extraction" (TRDE) for convenience.

\section{Experimental}

\subsection{Reagents and materials}

Water was purified with an Elix 3 UV system (Millipore Co., Billerica, MA). All reagents used were obtained commercially and were of analytical grade. Perylene, Eosin Y, acetonitrile, ethyl acetate, and chloroform were purchased from Wako Pure Chemical Industries, Ltd. (Osaka, Japan). 8-Hydroxyquinoline and an iron ion solution (1000 ppm, $0.275 \mathrm{M} \mathrm{HNO}_{3}$ ) were purchased from Nacalai Tesque, Inc. (Kyoto, Japan). A microchip made of glass incorporating microchannels was manufactured by Microchemical Technology (Kanagawa, Japan). Figure 1 illustrates the setup of the microchip in the microchip holder (Figure 1 (a)) and the enlarged view of the microchip incorporating the triple-branched microchannels (Figure 1 (b)). As shown in Figure 1 (b), one wide channel $(300 \mu \mathrm{m}$ wide $\times 45 \mu \mathrm{m}$ deep) denoted as channel $\mathrm{W}$ was separated into three narrow channels (each $100 \mu \mathrm{m}$ wide $\times 45 \mu \mathrm{m}$ deep) designated as channels N1-N3.

a)

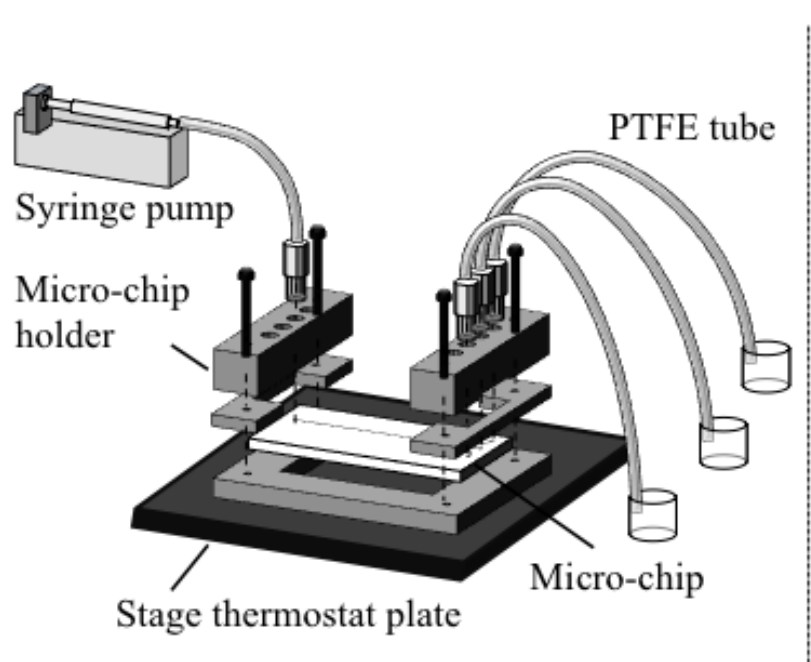

b)

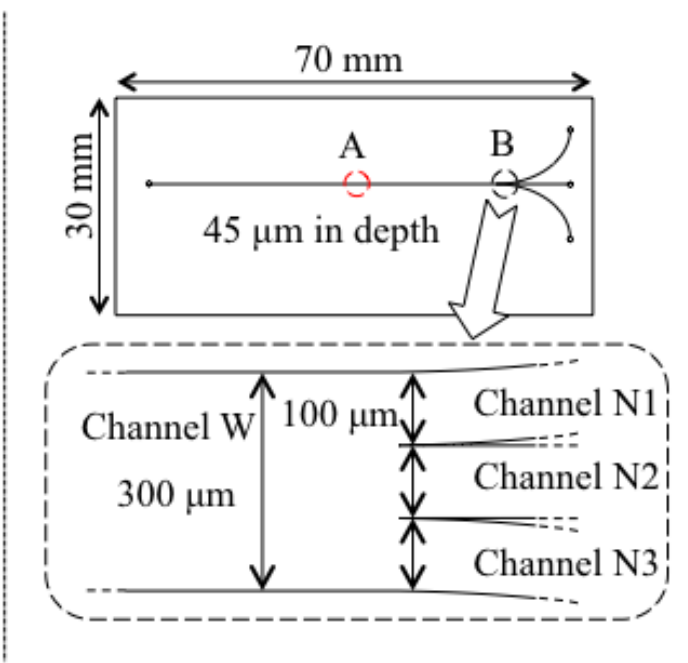

Figure 1. Illustration of the setup of a microchip in the microchip holder (a) and an enlarged view of the microchip incorporating the triple-branched microchannels (b). 


\subsection{Apparatus and procedures}

A microchip incorporating the microchannels was set up for the fluorescence microscope-CCD camera system. Fluorescence in the microchannel was monitored using a fluorescence microscope (BX51; Olympus, Tokyo, Japan) equipped with an Hg lamp, a filter (U-MWU2, ex. 330-385 nm, em. >420 nm), and a CCD camera (JK-TU53H). The ternary mixed solvents of water (acetic acid aqueous solution, pH3.5)-acetonitrile-ethyl acetate mixture and water (acetic acid aqueous solution, pH3.5)-acetonitrile-chloroform contained $0.1 \mathrm{mM}$ perylene and $1 \mathrm{mM}$ Eosin $\mathrm{Y}$. The solution was fed into the microchannels using a microsyringe pump. Acetic acid aqueous solution, $\mathrm{pH} 3.5$, was used in this study, and the solution was designated as just the water below.

The homogenous solution of the water-acetonitrile-ethyl acetate mixture (40:45:15 volume ratio) containing $5.0 \mu \mathrm{M} \mathrm{Fe}$ (III) and $10 \mathrm{mM}$ 8-hydroxyquinoline was fed into channel $\mathrm{W}$ at the defined flow rate and temperature $\left(100 \mu \mathrm{L} \min ^{-1}\right.$ and $\left.18^{\circ} \mathrm{C}\right)$ under the TRDP conditions. The solutions in channels N1-N3 were collected through polytetrafluoroethylene (PTFE) tubes into the corresponding vessels. The Fe(III) solutions were subjected to evaporation, and the resultant residues were dissolved in $0.1 \mathrm{M}$ nitric acid (10 $\mathrm{mL}$ ) for inductively coupled plasma atomic emission spectroscopy (ICP, 8100-Type instrument, Shimadzu Co., Kyoto, Japan).

\section{Results and Discussion}

\subsection{Two-phase separation in a batch vessel}

Homogeneous solutions of the ternary mixed solution separated into two distinct phases in a batch vessel when cooled below a certain temperature (i.e., the cloud point); a temperature-induced phase separation occurs $[10,11]$. One phase behaves as an organic solvent-rich solution (upper phase), whereas the other phase is a water-rich solution (lower phase). Figure 2 shows the two-phase separation system of thewater-acetonitrile-ethyl acetate mixed solvent solution containing $\mathrm{Fe}$ (III) and 8-hydroxyquinoline. Hydrophobic compounds dissolved in the homogeneous solution are extracted into the organic solvent-rich phase, whereas hydrophilic compounds remain in the aqueous phase. $\mathrm{Fe}(\mathrm{III})$ was extracted through 8-hydroxyquinoline complex formation with the ternary mixed solvent solution, water-acetonitrile-ethyl acetate, in a batch-type reactor as shown in Figure 2 [8].

\subsection{TRDP observation in the triple-branched microchannels}

The phase diagrams of the two ternary mixed solvent solutions, namely water-acetonitrile-ethyl acetate and water-acetonitrile-chloroform, are shown in Figure 3. The two ternary mixed solvent solutions

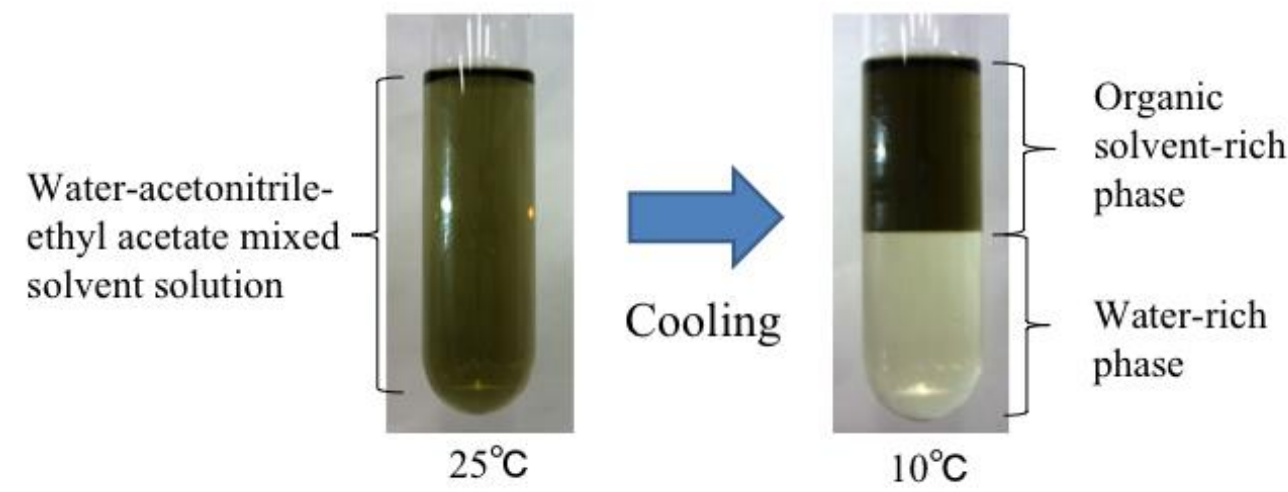

Figure 2. Two-phase separation system of the water-acetonitrile-ethyl acetate mixed solvent solution including $\mathrm{Fe}$ (III) and 8-hydroxyquinoline. 

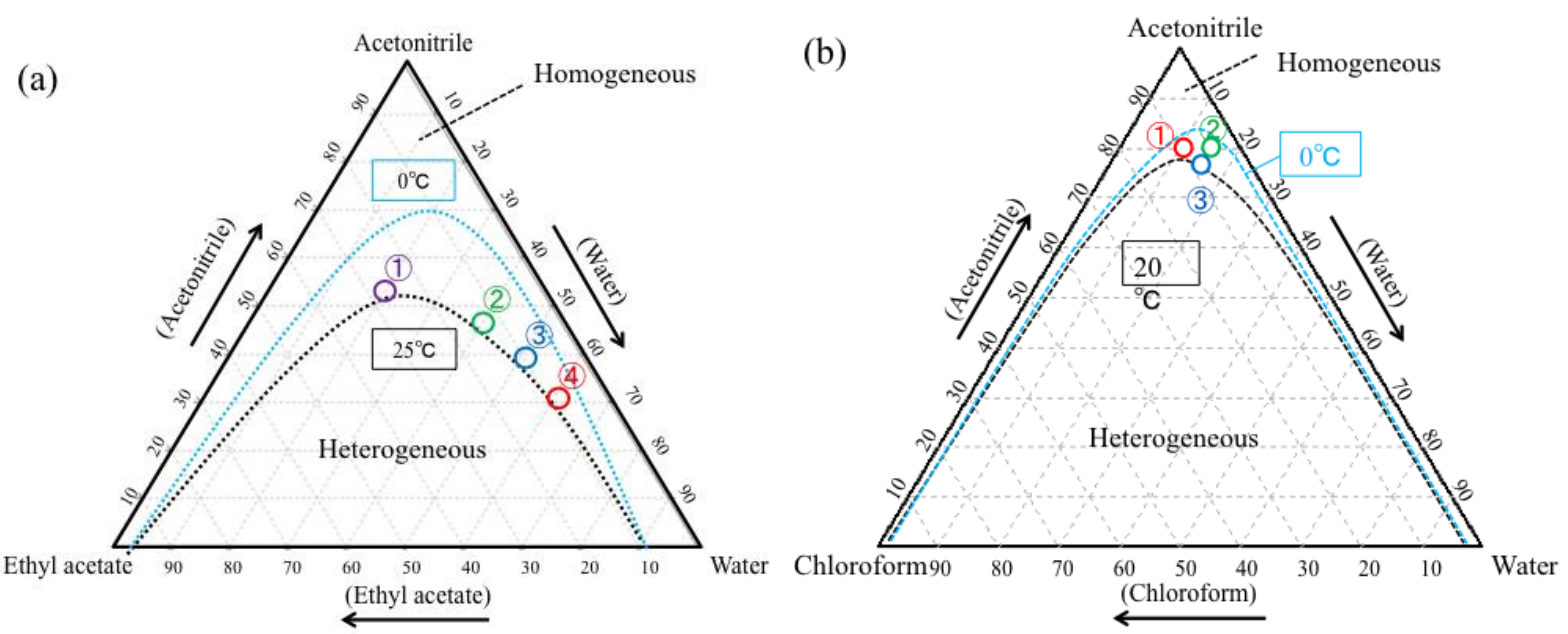

Figure 3. Phase diagrams of ternary mixed solvent solutions. The dotted curves show the boundary between the homogeneous and heterogeneous solutions at 0 and $25^{\circ} \mathrm{C}$. (a) Water-acetonitrile-ethyl acetate mixed solvent solution; the volume ratios of the ternary mixed solvent solutions, (1) 20:53:27, (2) 40:45:15, (3) 50:40:10, and (4) 60:30:10 and (b) water-acetonitrile-chloroform mixed solvent solution; the volume ratios of the ternary mixed solvent solutions, (1) 10:80:10, (2) 15:80:5, and (3) 15:75:10.

were applied as carrier solutions to the capillary chromatography based on the TRDP. This is called "tube radial distribution chromatography" (TRDC). The boundary curves between the homogeneous and heterogeneous solutions expanded in the phase diagrams by changing the temperature from $25^{\circ} \mathrm{C}$ to $0^{\circ} \mathrm{C}$.

The TRDP was examined in the microchip incorporating the triple-branched microchannels with the two ternary mixed solvent solutions through the fluorescence-CCD camera; water-acetonitrile-ethyl acetate mixtures of volume ratios of (1) $20: 53: 27$, (2) 40:45:15, (3) $50: 40: 10$, and (4) $60: 30: 10$ as well as water-acetonitrile-chloroform of volume ratios of (1)10:80:10, (2)15:80:5, and (3)15:75:10. The volume ratios are marked in the phase diagrams (Figure 3). The most stable TRDP in the triple-branched microchannels was focused to be the water-acetonitrile-ethyl acetate mixtures having the volume ratios of (2) 40:45:15. The obtained fluorescence photographs at the points A and B in the microchannel are shown in

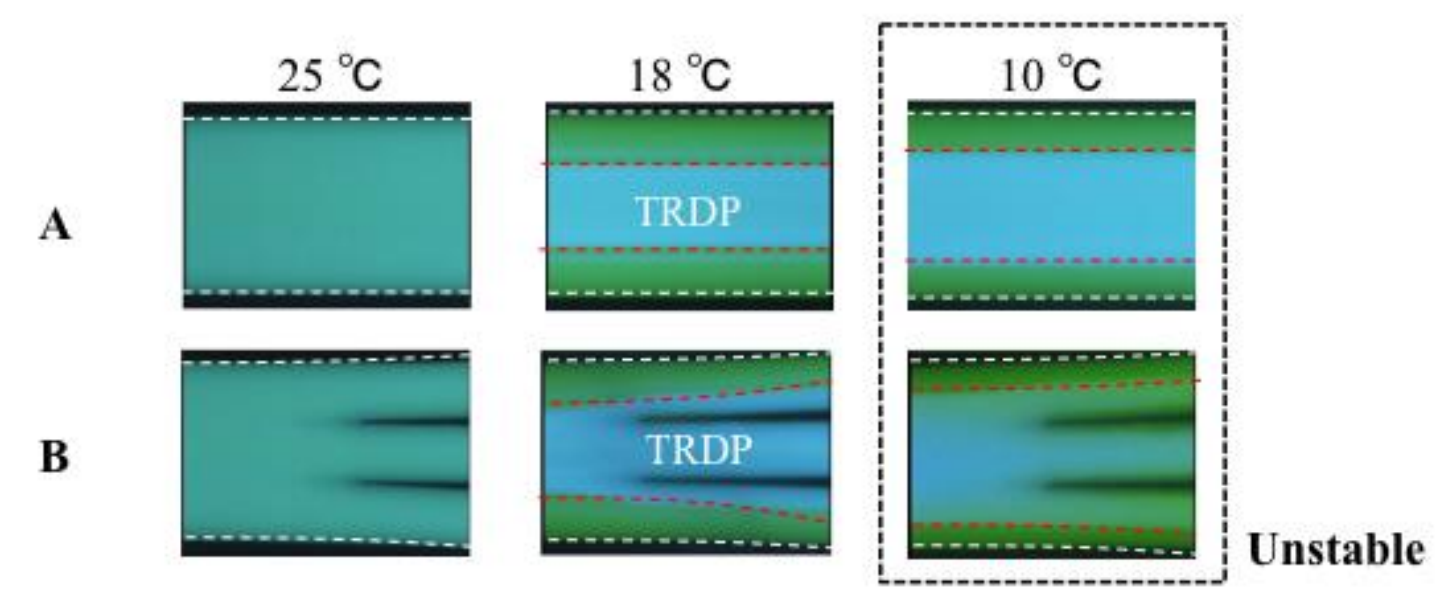

Figure 4. Fluorescent photographs of water-acetonitrile-ethyl acetate mixed solvent solution (40:45:15 volume ratio). Flow rate $20 \mu \mathrm{L} \mathrm{min}^{-1}$. The photographs were taken at points A and B in Figure 2. 


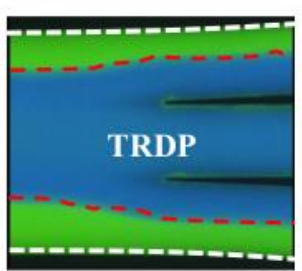

$2.0 \mu \mathrm{L} / \mathrm{min}$

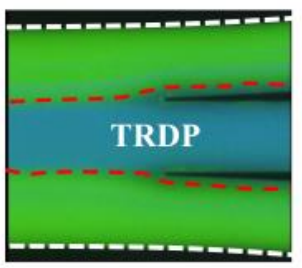

$50.0 \mu \mathrm{L} / \mathrm{min}$

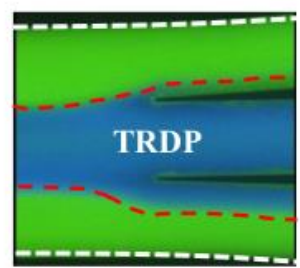

$10.0 \mu \mathrm{L} / \mathrm{min}$

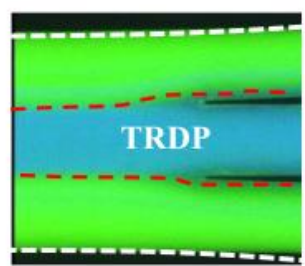

$100.0 \mu \mathrm{L} / \mathrm{min}$

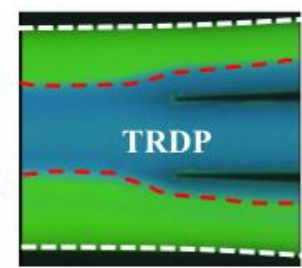

$20.0 \mu \mathrm{L} / \mathrm{min}$

Figure 5. Fluorescent photographs of water-acetonitrile-ethyl acetate mixed solvent solution (40:45:15 volume ratio) at various flow rates. $18^{\circ} \mathrm{C}$.

Figure 4 together with the temperature effects; non-TRDP or homogeneous microfluidic behavior, TRDP, and unstable TRDP [12] were respectively observed at $25{ }^{\circ} \mathrm{C}, 18^{\circ} \mathrm{C}$, and $10^{\circ} \mathrm{C}$. Also, the effects of flow rates $\left(2.0-100 \mu \mathrm{Lmin}^{-1}\right)$ were examined for the ternary water-acetonitrile-ethyl acetate mixed solvent solution (40:45:15 volume ratio) at $18^{\circ} \mathrm{C}$ (Figure 5). The microfluidic flow based on the TRDP well appeared along the triple-branched microchannel figures at 50 and $100 \mu \mathrm{Lmin}^{-1}$. Flow rate larger than 100 $\mu \mathrm{Lmin}^{-1}$ were not possible in the present experimental device. As mentioned above, we have observed TRDP only as a two-dimensional information. We are now trying to examine the distribution of fluorescent compounds at a cross-section of the microchannel with confocal microscopy to obtain three-dimensional information.

\subsection{Fe(III) distribution of based on TRDP in triple-branched microchannels}

From the above experimental data, the distribution of Fe(III) based on TRDP in the triple-branched microchannel was examined under the conditions of the water-acetonitrile-ethyl acetate mixed solvent (40:45:15 volume ratio), at $18^{\circ} \mathrm{C}$, and $100 \mu \mathrm{Lmin}^{-1}$. First, the homogenous solution of the ternary mixed solvent solution containing $17 \mu \mathrm{M} \mathrm{Fe}$ (III) and $10 \mathrm{mM}$ 8-hydroxyquinoline was fed into channel $\mathrm{W}$ at $25^{\circ} \mathrm{C}$ at a flow rate of $100 \mu \mathrm{L} \mathrm{min}^{-1}$. The solutions in the channels N1-N3 were collected via PTFE tubes into the corresponding vessels. Fe(III) concentrations in the three vessels were almost the same, around $17 \mu \mathrm{M}$, from the ICP analysis. No distribution of $\mathrm{Fe}(\mathrm{III})$ was naturally observed for the non-TRDP system at $25^{\circ} \mathrm{C}$. Next, the same ternary mixed solvent solution was similarly delivered into channel $\mathrm{W}$ at $18^{\circ} \mathrm{C}$, and the $\mathrm{Fe}$ (III) concentrations in the vessels were examined. The Fe(III) concentrations in the center wide channel (channel N2) and the two side channels (channels N1 and N3) were 10, 28, and $11 \mu \mathrm{M}$, respectively (Figure 6). The experimental data indicated that $\mathrm{Fe}(\mathrm{III})$ was distributed or extracted to the organic solvent-rich solution in the center narrow channel from the homogeneous solution by Fe(III)-8-hydroxyquinoline complex formation. Also, extraction of $\mathrm{Fe}$ (III) was carried out with a batch vessel under similar conditions (cooling and standing for $30 \mathrm{~min}$ ), and $\mathrm{Fe}(\mathrm{III})$ was distributed in a ratio of 65:35 to the organic solvent-rich upper phase and the water-rich lower phase. We need to more strictly separate the two phases (organic solvent-rich and water-rich) in the microchannel. 


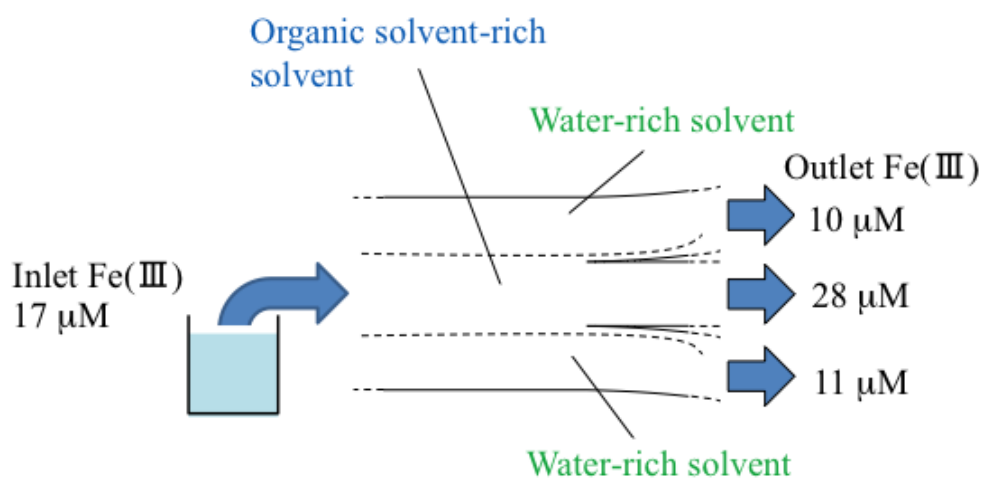

Figure 6. Extraction of $\mathrm{Fe}(\mathrm{III})$ from the homogeneous ternary mixed solution into the inner phase in the center narrow channel through TRDP. Conditions: Carrier, water-acetonitrile-ethyl acetate (40:45:15, v/v/v) mixture; flow rate, $100 \mathrm{~mL} \mathrm{~min}^{-1}$ for channel W; initial $\mathrm{Fe}(\mathrm{III})$ concentration, $17 \mu \mathrm{M}$ and 8-hydroxyquinoline, $10 \mu \mathrm{M}$.

\section{Conclusions}

A microflow extraction process with complexing reagents was demonstrated using a microchip incorporating triple-branched microchannels for the specific microfluidic behavior of the TRDP. Fe(III) as a model was dissolved in the homogenous solution of the water-acetonitrile-ethyl acetate mixed solvent solution containing 8-hydroxyquinoline. The solution was fed into the microchannels to generate the inner and outer phases through the TRDP under laminar flow conditions. The Fe(III)-8-hydroxyquinoline complex was distributed between the inner and outer phases and then was extracted to the central narrow channel due to its hydrophilic nature. We call the extraction procedure "tube radial distribution extraction" (TRDE).

\section{Acknowledgement}

This work was supported by a Grant-in-Aid for Scientific Research (C) from the Ministry of Education, Culture, Sports, Science, and Technology (MEXT), Japan.

\section{References}

1) F. Wang, W. Bi, Q. Sun, Sensors \& Transducers J., 166, 29-38 (2014).

2) E. Y. Basova, F. Foret, Analyst, 140, 22-38 (2015).

3) A. B. Vir, A. S. Fabiyan, J. R. Picardo, S. Pushpavanam, Ind. Eng. Chem. Res., 53, 8171-8181 (2014).

4) M. Maeki, Y. Hatanaka, K. Yamashita, M. Miyazaki, K. Ohto, Solvent. Extr. Res. Dev., Jpn., 21, 77-82 (2014).

5) N. Jinno, M. Murakami, K. Mizohata, M. Hashimoto, K. Tsukagoshi, Analyst, 135, 927-932 (2011).

6) M. Murakami, N. Jinno, M. Hashimoto, K. Tsukagoshi, Anal. Sci., 27, 793-798 (2011).

7) K. Tsukagoshi, Anal. Sci., 30, 65-73 (2014), and references cited therein.

8) N. Takahashi, M. Hashimoto, K. Tsikagoshi, Anal. Sci., 29, 665-667 (2013).

9) N. Jinno, M. Hashimoto, K. Tsukagoshi, J. Anal. Sci., Methods and Instrumentation, 2, 49-53 (2012).

10) K. Fujinaga, Anal. Sci., 9, 479-482 (1993).

11) P. I. Trindade, M. M. Diogo, D. M. F. Prazeres, C. J. Marcos, J. Chromatogr. A, 1082, 176-184 (2005).

12) S. Fujinaga, M. Hashimoto, K. Tsukagoshi, J. Chem. Eng. Japan, in press 